Rev. Mat. Iberoamericana 20 (2004), 531-561

\title{
On the product theory of singular integrals
}

\section{Alexander Nagel and Elias M. Stein}

\begin{abstract}
We establish $L^{p}$-boundedness for a class of product singular integral operators on spaces $\widetilde{M}=M_{1} \times M_{2} \times \cdots \times M_{n}$. Each factor space $M_{i}$ is a smooth manifold on which the basic geometry is given by a control, or Carnot-Carathéodory, metric induced by a collection of vector fields of finite type. The standard singular integrals on $M_{i}$ are non-isotropic smoothing operators of order zero. The boundedness of the product operators is then a consequence of a natural LittlewoodPaley theory on $\widetilde{M}$. This in turn is a consequence of a corresponding theory on each factor space. The square function for this theory is constructed from the heat kernel for the sub-Laplacian on each factor.
\end{abstract}

\section{Introduction}

The purpose of this paper is to develop an $L^{p}$ theory of product singular integrals in sufficient generality so that it can be used in a number of different situations, and in particular for estimates of fundamental solutions of $\square_{b}$ on certain model domains in several complex variables. These applications will be described in [NS03]. Beyond these applications, this theory would seem to have an interest in its own right, as it differs in its setting from the product theory studied in earlier papers. We now describe this background.

Any analysis of product singular integrals on a product space

$$
\widetilde{M}=M_{1} \times M_{2} \times \cdots \times M_{n}
$$

must be based, to start with, on a formulation of standard singular integrals on each factor $M_{i}$. Such a class of operators on $M_{i}$ can in turn be defined in

2000 Mathematics Subject Classification: Primary 42B20, 42B25.

Keywords: product singular integrals, control metrics, NIS operators, Littlewood-Paley theory. 
relation to the underlying geometric structure of each $M_{i}$. As a first example, take the factors $M_{i}$ to be Euclidean spaces, and the class of operators on $M_{i}$ to be the convolution operators of (loosely speaking) Calderón-Zygmund type. Then a corresponding product theory is developed in [FS82], but its roots go back, at least implicitly, to [JMZ35]. A generalization to the case where the $M_{i}$ are appropriate nilpotent groups and the operators are of convolution type is carried out in [MRS95] and [NRS01].

For the applications we have in mind, we need to generalize this situation and replace each $M_{i}$ by a space of more general type: one with a geometry determined by a control distance defined in terms of a collection of distinguished vector fields $\left\{X_{1}, X_{2}, \ldots, X_{k}\right\}$ having the property that they and their commutators span the tangent space at each point.

There are now at least two paths open to us. One is to generalize the class of operators on each factor $M_{i}$ to the extended class of the $T(1)$ theorem of David and Journé [DJ84], and then pass from this to a corresponding product theory. This idea was carried out in [Jou85] in the setting where each factor is a Euclidean space.

One might be able to carry out this approach for more general $M_{i}$. However, because of the inherent complications, we choose a second, simpler approach. We develop a theory which is more tractable and directly applicable on the product $\widetilde{M}$ even though the theory on each factor is somewhat less general in scope. Specifically, we consider on each factor an intermediate degree of generality- the class of singular integrals of NIS type (non-isotropic smoothing operators of order 0 ). These operators occur naturally on the boundary of various domains in $\mathbb{C}^{n}$ (see [NRSW89], [CNS92], and [Koe02]). They may be viewed as Calderón-Zygmund operators whose kernels are $C^{\infty}$ away from the diagonal, and whose cancellation conditions are given quite simply in terms of their action on smooth bump functions. It is a combination of these two properties that make the operators on each $M_{i}$ easy to handle, and this carries over to the product-type operators on $\widetilde{M}$.

The precise definition of the class of operators on each $M_{i}$ is given below in section 2. Any product theory tends to be burdened with notational complexities. It seems easiest to first present full details of the product kernels when there are only two factors, and then consider the general situation. Thus the definition of the corresponding class of product operators for two factors is given in section 3, and the definition of the class of operators on an arbitrary finite Cartesian product is given in section 4 .

The key to the proof of the $L^{2}$ and $L^{p}$ boundedness of these operators is the existence of a natural Littlewood-Paley theory on $\widetilde{M}$, which is itself a consequence of the corresponding theory on each factor. The square function that we use is constructed in terms of the heat equation, and the 
details of this are carried out in section 2. The basic interplay between the singular integrals and the square function is provided by Proposition 3.3.1 in the single-factor case, and Proposition 4.2.4 in the general product case. Our main theorems, (Theorems 4.1.2 and 5.1.1), on the $L^{p}$ boundedness of product operators then follows easily from these considerations.

\section{The Littlewood-Paley Theory}

We are interested in demonstrating the $L^{p}$ boundedness of product singular integrals on a product space $\widetilde{M}=M_{1} \times M_{2} \times \cdots \times M_{n}$. To carry this out we shall first need to describe a Littlewood-Paley theory for each factor $M_{i}$, $1 \leq i \leq n$, and then pass to the corresponding product theory. We begin by concentrating on a fixed $M_{i}$, which we denote by $M$, dropping the subscript $i$. We describe the corresponding underlying geometric structure, and then the resulting square functions defined in terms of the heat equation.

For the sake of simplicity, and because of the applications we have in mind (see [NS03]), we shall focus our attention on two specific settings:

(A) Here $M$ is a compact connected $C^{\infty}$-manifold. We suppose that we are given $k$ smooth real vector fields on $\left\{X_{1}, \ldots, X_{k}\right\}$ on $M$ which are of finite-type $m$ in the sense that these vector fields together with their commutators of order $\leq m$ span the tangent space to $M$ at each point.

(B) Here $M$ arises as the boundary of an unbounded model polynomial domain in $\mathbb{C}^{2}$. Thus let $\Omega=\left\{(z, w) \in \mathbb{C}^{2} \mid \Im \mathrm{m}[w]>P(z)\right\}$, where $P$ is a real, subharmonic, non-harmonic polynomial of degree $m$. Then $M=$ $\partial \Omega$ can be identified with $\mathbb{C} \times \mathbb{R}=\{(z, t), z \in \mathbb{C}, t \in \mathbb{R}\}$. The basic $(0,1)$ Levi vector field is then $\bar{Z}=\frac{\partial}{\partial \bar{z}}-i \frac{\partial P}{\partial \bar{z}} \frac{\partial}{\partial t}$, and we write $\bar{Z}=X_{1}+$ $i X_{2}$. The real vector fields $\left\{X_{1}, X_{2}\right\}$ and their commutators of orders $\leq m$ span the tangent space at each point. Thus this $M$ is a special non-compact variant, with $k=2$, of the manifolds considered in (A).

\subsection{Geometry on $M$}

The most important geometric object we need is one of a class of equivalent control distances constructed on $M$ via the vector fields $\left\{X_{1}, \cdots X_{k}\right\}$. One variant of the control distance can be defined as follows. For $x, y \in M$, let $A C(x, y, \delta)$ denote the collection of absolutely continuous mappings $\varphi$ : $[0,1] \rightarrow M$ with $\varphi(0)=x, \varphi(1)=y$, and for almost every $t \in[0,1]$, $\varphi^{\prime}(t)=\sum_{j=1}^{k} a_{k} X_{k}(\varphi(t))$ with $\left|a_{k}\right| \leq \delta$. Then the control distance $\rho(x, y)$ from $x$ to $y$ is the infimum of the set of $\delta>0$ such that $A C(x, y, \delta) \neq \emptyset$. For details, see [NSW85], and [NS01b]. 
The result we need here is that there is a pseudo-metric $d \approx \rho$ on $M$ equivalent to this control metric ${ }^{1}$ which has the optimal smoothness; i.e. $d(x, y)$ is $C^{\infty}$ on $\{M \times M-$ diagonal $\}$, and for $x \neq y$

$$
\left|\partial_{X}^{K} \partial_{Y}^{L} d(x, y)\right| \lesssim d(x, y)^{1-K-L} .
$$

(Here $\partial_{X}^{K}$ is a product of $K$ of the vector fields $\left\{X_{1}, \ldots, X_{k}\right\}$ acting as derivatives on the $x$-variable, and $\partial_{Y}^{L}$ are a corresponding $L$ vector fields acting on the $y$-variable). For the existence of such a pseudo-metric, see Theorems 3.3.1 and 4.4.6 in [NS01b] where $d$ is denoted by $\tilde{\rho}$. We shall also assume that in the case (A), upon using an equivalent pseudo-metric, we have $d(x, y) \leq 1$ for all $x, y \in M$.

We consider a volume measure on $M$ as follows. When we are in the compact situation (A), then we take any fixed smooth measure on $M$ with strictly positive density. In the situation (B) we take Lebesgue measure on $\mathbb{C} \times \mathbb{R}$. In either case, when integrating we write the measure as $d x$. We denote the measure of a set $E$ by $|E|$.

We define balls $B(x, \delta)=\{y \in M, d(x, y)<\delta\}$, with $0<\delta \leq 1$ in case (A), and $0<\delta<\infty$ in case (B). We have the following formulae for the volume $|B(x, \delta)|$ :

$$
\begin{array}{ll}
|B(x, \delta)| \approx \sum_{|I| \leq r}\left|\lambda_{I}(x)\right| \delta^{|I|} & \text { in case }(\mathrm{A}) \\
|B(x, \delta)| \approx\left(\sum_{k=2}^{m} \Lambda_{k}(x) \delta^{k}\right) \delta^{2} & \text { in case }(\mathrm{B}) .
\end{array}
$$

Here $\left|\lambda_{I}\right|$ and $\Lambda_{k}$ are the appropriate Levi-invariants, and are continuous, non-negative functions on $M$ (see theorem 2.2.4 and section 4.1 in [NS01b]). The balls have the required doubling property

$$
|B(x, 2 \delta)| \leq C|B(x, \delta)|, \text { for all } \delta>0 .
$$

We also introduce the volume functions

$$
\begin{aligned}
V_{\delta}(x) & =|B(x, \delta)|, \\
V(x, y) & =|B(x, d(x, y))| .
\end{aligned}
$$

It is important to observe that

$$
V(x, y) \approx V(y, x)
$$

\footnotetext{
${ }^{1}$ Here, and throughout the paper, $A \approx B$ means that the ratio $A / B$ is bounded and bounded away from zero by constants that do not depend on the relevant variables in $A$ and $B . A \lesssim B$ means that the ratio $A / B$ is bounded by a constant independent of the relevant variables. We shall also assume that the pseudo-metric satisfies $d(x, y)=d(y, x)$.
} 
In fact if $d(x, y) \leq \delta$, then $B(y, \delta) \subset B(x, C \delta)$, so $|B(y, \delta)| \leq|B(x, C \delta)| \leq$ $C^{\prime}|B(x, \delta)|$ by the doubling property. If we take $\delta=d(x, y)$, this gives $V(y, x) \leq C^{\prime} V(x, y)$, and by symmetry (2.6) is established. Also note that in case (B), it follows from (2.3) that we have

$$
|B(x, s \delta)| \geq s^{4}|B(x, \delta)| \quad \text { for } s \geq 1 .
$$

Remark 2.1.1 Whenever $a>0$, there is a constant $C_{a}$ so that

$$
\int_{d(x, y) \leq 1} \frac{d(x, y)^{+a}}{V(x, y)} d y \leq C_{a}, \quad \text { and } \quad \int_{d(x, y) \geq 1} \frac{d(x, y)^{-a}}{V(x, y)} d y \leq C_{a} .
$$

In fact we can write the first integral as

$$
\sum_{k=0}^{\infty} \int_{2^{-k-1}<d(x, y) \leq 2^{-k}} \frac{d(x, y)^{a}}{V(x, y)} d y .
$$

On the other hand,

$$
\begin{aligned}
\int_{2^{-k-1}<d(x, y) \leq 2^{-k}} \frac{d(x, y)^{a}}{V(x, y)} d y & \leq 2^{-k a}\left|B\left(x, 2^{-k-1}\right)\right|^{-1} \int_{d(x, y) \leq 2^{-k}} d y \\
& =2^{-k a} \frac{\left|B\left(x, 2^{-k}\right)\right|}{\left|B\left(x, 2^{-k-1}\right)\right|} \leq C 2^{-k a} .
\end{aligned}
$$

Summing in $k$ gives the estimate for the first integral. The second integral in (2.8) is non-zero only in case (B) and is dealt with in the same way.

\subsection{The maximal function}

The analogue of the standard maximal function is defined as follows:

$$
\mathcal{M}[f](x)=\sup _{\delta>0} \frac{1}{|B(x, \delta)|} \int_{B(x, \delta)}|f(y)| d y .
$$

Note that for $a>0$,

$$
\sup _{\delta>0} \delta^{a} \int_{d(x, y)>\delta} \frac{(d(x, y))^{-a}}{V(x, y)}|f(y)| d y \leq C \mathcal{M}[f](x) .
$$

In fact,

$$
\begin{aligned}
& \delta^{a} \int_{2^{k+1} \delta} \geq d(x, y)>2^{k} \delta \frac{d(x, y)^{-a}}{V(x, y)}|f(y)| d y \\
& \leq 2^{-k a} \frac{1}{\left|B\left(x, 2^{k} \delta\right)\right|} \int_{\left|B\left(x, 2^{k+1} \delta\right)\right|}|f(y)| d y \leq C 2^{-k a} \mathcal{M}[f](x)
\end{aligned}
$$

and summing in $k$ establishes (2.9). 
Standard arguments (see [Ste93, Chapter I]) using the properties of the balls, and in particular the doubling property (2.4), prove that

$$
\|\mathcal{M}[f]\|_{L^{p}(M)} \leq A_{p}\|f\|_{L^{p}(M)}, \quad 1<p \leq \infty .
$$

We shall also need a vector-valued form of this inequality, which we formulate in its continuous version. Suppose $f(s, x)=f_{s}(x)$ is a measurable function on $\mathbb{R}^{+} \times M$ so that $f_{s} \in L^{p}(M)$ for almost every $s$. Then for $1<p<\infty$ there is a constant $A_{p}$ so that

$$
\left\|\left(\int_{0}^{\infty} \mathcal{M}\left(f_{s}\right)^{2} d s\right)^{1 / 2}\right\|_{L^{p}(M)} \leq A_{p}\left\|\left(\int_{0}^{\infty}\left|f_{s}\right|^{2} d s\right)^{1 / 2}\right\|_{L^{p}(M)}
$$

The proof of (2.11) follows as in [Ste93, Chapter II, Section 1].

\subsection{The Heat Equation}

We consider the sub-Laplacian $\mathcal{L}$ on $M$ in self-adjoint form, given by

$$
\mathcal{L}=\sum_{j=1}^{k} X_{j}^{*} X_{j}
$$

Here $\left(X_{j}^{*} \varphi, \psi\right)=\left(\varphi, X_{j} \psi\right)$, where

$$
(\varphi, \psi)=\int_{M} \varphi(x) \overline{\psi(x)} d x
$$

and $\varphi, \psi \in C_{0}^{\infty}(M)$, the space of $C^{\infty}$ functions on $M$ of compact support. In general, $X_{j}^{*}=-X_{j}+a_{j}$ where $a_{j} \in \mathcal{C}^{\infty}(M)$.

We are interested in the initial value problem for the heat equation:

$$
\frac{\partial u}{\partial s}(x, s)+\mathcal{L}_{x} u(x, s)=0, \text { with } u(x, 0)=f(x) .
$$

The solution we have in mind is given by

$$
u(x, s)=H_{s}[f](x),
$$

where $H_{s}$ is the operator given via the spectral theorem by $H_{s}=e^{-s \mathcal{L}}$, and an appropriate self-adjoint extension of the non-negative operator $\mathcal{L}$ initially defined on $C_{0}^{\infty}(M)$. (See [NS01a], and in particular the remarks preceding Theorem 2.3.5). One can then assert: 
Proposition 2.3.1 Whenever $f \in L^{2}(M)$, we have

$$
H_{s}[f](x)=\int_{M} H(s, x, y) f(y) d y .
$$

Moreover

1. $H(s, x, y) \in C^{\infty}([0, \infty) \times M \times M \backslash\{s=0$ and $x=y\})$. For $s>0$,

$$
\frac{\partial H}{\partial s}(s, x, y)=-\mathcal{L}_{x} H(s, x, y)=-\mathcal{L}_{y} H(s, x, y) .
$$

2. $H(s, x, y)$ satisfies the following differential inequalities for every integer $N \geq 0$ :

(a) When $d^{2}(x, y) \geq s$,

$$
\left|\partial_{s}^{j} \partial_{X}^{L} \partial_{Y}^{K} H(s, x, y)\right| \lesssim \frac{d(x, y)^{-2 j-K-L}}{V(x, y)}\left(\frac{s}{d^{2}(x, y)}\right)^{N}
$$

(b) When $d^{2}(x, y) \leq s$,

$$
\left|\partial_{s}^{j} \partial_{X}^{L} \partial_{Y}^{K} H(s, x, y)\right| \lesssim \frac{s^{-j-K / 2-L / 2}}{V_{\sqrt{s}}(x)} .
$$

In particular, for every $L$ and $K$, there is a constant $C_{L, K}$ so that for all $s \in(0, \infty)$ and all $x, y \in M$

$$
\left|\partial_{X}^{L} \partial_{Y}^{K} H(s, x, y)\right| \leq C_{K, L} \frac{d(x, y)^{-K-L}}{V(x, y)} .
$$

3. For each integer $L \geq 0$ there exists an integer $N_{L}$ and a constant $C_{L}$ so that if $\varphi \in \mathcal{C}_{0}^{\infty}\left(B\left(x_{0}, \delta\right)\right)$, then for all $s \in(0, \infty)$

$$
\left|\partial_{X}^{L} H_{s}[\varphi]\left(x_{0}\right)\right| \leq C_{L} \delta^{-L} \sup _{x} \sum_{|J| \leq N_{L}} \delta^{|J|}\left|\partial_{X}^{J} \varphi(x)\right| .
$$

4. For all $(s, x, y) \in(0, \infty) \times M \times M$,

$$
\begin{aligned}
& H(s, x, y)=H(s, y, x) \\
& H(s, x, y) \geq 0 .
\end{aligned}
$$

5. For all $(s, x) \in(0, \infty) \times M, \int_{M} H(s, x, y) d y=1$.

6. For $1 \leq p \leq \infty,\left\|H_{s}(f)\right\|_{L^{p}(M)} \leq\|f\|_{L^{p}(M)}$.

7. For every $\varphi \in \mathcal{C}_{0}^{\infty}(M)$ and every $t \geq 0$,

$$
\lim _{s \rightarrow 0}\left\|H_{s}[\varphi]-\varphi\right\|_{t}=0
$$

where $\|\cdot\|_{t}$ denotes the Sobolev norm. 
Proof. The assertions (1), (2), and (3) of Proposition 2.3.1 are proved in [NS01a]. In establishing (4), the fact that $H(s, x, y)$ is real follows easily from the fact that the operator $\mathcal{L}$ is real, and the symmetry $H(s, x, y)=$ $H(s, y, x)$ is then a consequence of the fact that $e^{-s \mathcal{L}}$ is self-adjoint.

The proof of the non-negativity of $H(s, x, y)$ requires several steps. First, since $\mathcal{L}$ is non-negative, for each $\lambda>0$ the resolvent

$$
R(\lambda, \mathcal{L})=(\lambda I+\mathcal{L})^{-1}
$$

is a bounded operator on $L^{2}(M)$ with norm $\leq 1 / \lambda$ by the spectral theorem. Notice also that

$$
(\lambda I+\mathcal{L})^{-1}[f]=\lim _{\epsilon \rightarrow 0} \int_{\epsilon}^{1 / \epsilon}\left(e^{-\lambda s} e^{-s \mathcal{L}}\right)[f] d s=\lim _{\epsilon \rightarrow 0} \int_{\epsilon}^{1 / \epsilon} e^{-\lambda s} H_{s}[f] d s
$$

with the limit taken in the $L^{2}(M)$ norm. We shall need the following properties of $R(\lambda, \mathcal{L})$.

\section{Lemma 2.3.2}

(a) For $\lambda>0$ there is a constant $C_{\lambda}$ so that for all $f \in L^{2} \cap L^{\infty}$,

$$
\|R(\lambda, \mathcal{L})[f]\|_{L^{\infty}(M)} \leq C_{\lambda}\|f\|_{L^{\infty}(M)} .
$$

(b) If $f \in C_{0}^{\infty}(M)$, then $R(\lambda, \mathcal{L})[f] \in C^{\infty}(M)$.

(c) Let $C$ denote the space of continuous functions on $M$ vanishing at infinity. Then if $f \in L^{2} \cap C$, it follows that $R(\lambda, \mathcal{L})[f] \in L^{2} \cap C$.

(d) If $f \in L^{2}$ and $f \geq 0$, then $R(\lambda, \mathcal{L})[f] \geq 0$.

Proof of Lemma 2.3.2 Let us write

$$
R(\lambda, \mathcal{L})[f](x)=\int_{M} f(y) r_{\lambda}(x, y) d y .
$$

Then

$$
r_{\lambda}(x, y)=\int_{0}^{\infty} e^{-\lambda s} H(s, x, y) d s .
$$

We make two estimates on $r_{\lambda}(x, y)$. First, we claim that there is a constant $C$ so that for all $\lambda>0$ we have

$$
\left|r_{\lambda}(x, y)\right| \leq C \frac{d^{2}(x, y)}{V(x, y)}
$$

Second, for each $\lambda>0$ we claim that there is a constant $C_{\lambda}$ so that

$$
\left|r_{\lambda}(x, y)\right| \leq C_{\lambda} \frac{d^{-2}(x, y)}{V(x, y)}
$$

In the compact case (A), only the estimate (2.12) is relevant. 
To establish (2.12), we make no use of the decay given by the term $e^{-\lambda s}$. In fact using

$$
|H(s, x, y)| \lesssim \begin{cases}V(x, y)^{-1} & \text { when } s \leq d(x, y)^{2} \\ V_{\sqrt{s}}(x)^{-1} & \text { when } s \geq d(x, y)^{2}\end{cases}
$$

we see that

$$
\left|r_{\lambda}(x, y)\right| \leq\left(\int_{0}^{d(x, y)^{2}} d s\right) V(x, y)^{-1}+\int_{d(x, y)^{2}}^{\infty} V_{\sqrt{s}}(x)^{-1} d s .
$$

The second integral arises only in the noncompact case (B). To handle it, substitute $\sqrt{s}=d(x, y) t$. Then

$$
\int_{d(x, y)^{2}}^{\infty} V_{\sqrt{s}}(x)^{-1} d s=2 d(x, y)^{2} \int_{1}^{\infty} V_{d(x, y) t}(x)^{-1} t d t .
$$

But for $t \geq 1$, since we are dealing with case (B) it follows from (2.7) and (2.5) that

$$
V_{d(x, y) t}(x) \geq t^{4} V_{d(x, y)}(x)=t^{4} V(x, y) .
$$

Thus

$$
\int_{d^{2}(x, y)}^{\infty} V_{\sqrt{s}}(x)^{-1} d s \lesssim d(x, y)^{2} V(x, y)^{-1} \int_{1}^{\infty} u^{-3} d u \lesssim \frac{d^{2}(x, y)}{V(x, y)} .
$$

This gives the estimate (2.12).

To establish (2.13) we use the decay of $e^{-\lambda s}$, and the estimate

$$
|H(s, x, y)| \lesssim V(x, y)^{-1} \frac{s}{d(x, y)^{2}}
$$

when $s \leq d(x, y)^{2}$. We get

$$
\left|r_{\lambda}(x, y)\right| \lesssim\left(\int_{0}^{\infty} s e^{-\lambda s} d s\right) V(x, y)^{-1} d(x, y)^{-2}+\int_{d(x, y)^{2}}^{\infty} e^{-\lambda s} V_{\sqrt{s}}(x)^{-1} d s .
$$

The second term is dominated by

$$
\left(\int_{d(x, y)^{2}}^{\infty} s e^{-\lambda s} d s\right) V(x, y)^{-1} d(x, y)^{-2}
$$

and so we obtain

$$
\left|r_{\lambda}(x, y)\right| \lesssim \frac{d^{-2}(x, y)}{V(x, y)} .
$$

Thus (2.12) and (2.13) are established. 
Now using (2.12) when $d(x, y) \leq 1$, and (2.13) when $d(x, y) \geq 1$, we see that because of (2.8) we have

$$
\int_{M}\left|r_{\lambda}(x, y)\right| d y \leq C_{\lambda}
$$

which leads to conclusion (a) of Lemma 2.3.2.

Next, if $f \in C_{0}^{\infty}(M)$, then $f$ belongs to the domain of $\mathcal{L}^{N}$ for every positive integer $N$, and hence by the spectral theorem $\mathcal{L}^{N} R(\lambda, \mathcal{L}) f=$ $R(\lambda, f) \mathcal{L}^{N} f$. Since $\mathcal{L}$ is sub-elliptic it follows that $R(\lambda, \mathcal{L}) f \in C^{\infty}$, which establishes conclusion (b) of Lemma 2.3.2.

Notice that in the case (B) when $M$ is not compact, if $f \in \mathcal{C}_{0}^{\infty}(M)$ the estimates $(2.12)$ and $(2.13)$ show that $R(\lambda, \mathcal{L}) f$ vanishes at infinity. Assertion (c) of Lemma 2.3.2 is then a consequence of an approximation argument in the sup norm, using conclusion (a).

Finally, we turn to the crucial conclusion (d) regarding the positivity of $R(\lambda, \mathcal{L})$. It suffices to prove that $R(\lambda, \mathcal{L})[f] \geq 0$, whenever $f \in \mathcal{C}_{0}^{\infty}(M)$ and $f \geq 0$. Let $F=R(\lambda, \mathcal{L})[f]$. We have seen that $F$ is $\mathcal{C}^{\infty}$, and vanishes at infinity. Suppose that for some $x_{0} \in M, F\left(x_{0}\right)<0$; we shall show this leads to a contradiction. We may take $x_{0}$ to be a local minimum of $F$. Now $(\lambda I+\mathcal{L}) F=f$ and hence $\mathcal{L}(F)\left(x_{0}\right)=f\left(x_{0}\right)-\lambda F\left(x_{0}\right)$. Since $\lambda>0$ this implies

$$
\sum_{j=1}^{k}\left(X_{j}^{*} X_{j}\right)(F)\left(x_{0}\right)>0
$$

and hence for at least one $j, X_{j}^{*} X_{j}(F)\left(x_{0}\right)>0$. However, $X_{j}^{*}(f)=$ $-X_{j}(f)+a_{j} f$, and $X_{j}(F)\left(x_{0}\right)=0$, so it follows that $X_{j}^{2}(F)\left(x_{0}\right)<0$. Let $\gamma(t)=F\left(\left(\exp t X_{j}\right)\left(x_{0}\right)\right)$. Then $\gamma^{\prime}(0)=0$, while $\gamma^{\prime \prime}(0)<0$, contradicting the fact that $\gamma(t)$ has a local minimum at $t=0$. This completes the proof of Lemma 2.3.2.

We can now prove assertion (4) of Proposition 2.3.1. Since $R(\lambda, \mathcal{L})$ preserves $L^{2}$ and positivity, we see that the same holds for $(R(\lambda, \mathcal{L}))^{N}$, for every positive integer $N$. However if $s>0$, then

$$
\left(\frac{N}{s} R(N / s, \mathcal{L})\right)^{N} f=(I+(s / N) \mathcal{L})^{-N} f
$$

and by the spectral theorem this converges in the $L^{2}(M)$ norm to $e^{-s \mathcal{L}}(f)=$ $H_{s}(f)$; thus we see that $H_{s}$ is positivity preserving.

To prove assertion (5) of Proposition 2.3.1, let

$$
u(s, x)=\int_{M} H(s, x, y) d y .
$$

Note that this integral converges by the estimates (2) in Proposition 2.3.1, and those in (2.13). 
We show first that $\frac{\partial u}{\partial s}(s, x) \equiv 0$. Let

$$
u_{\sigma}(s, x)=\int_{M} H(s, x, y) \eta\left(\frac{d(x, y)}{\sigma}\right) d y
$$

where $\eta$ is a $C^{\infty}$ function with $\eta(u)=1$, if $u \leq 1 / 2$, and $\eta(u)=0$, if $u \geq 1$. Then by the estimates alluded to, the relevant integrals converge and this shows that $u_{\sigma}(s, x) \rightarrow u(s, x)$ and $\frac{\partial u_{\sigma}}{\partial s}(s, x) \rightarrow \frac{\partial u}{\partial s}(s, x)$ as $\sigma \rightarrow \infty$.

However, since $\eta\left(\frac{d(x, y)}{\sigma}\right)$ is in $C_{0}^{\infty}$ (as a function of $y$ ), we have that

$$
\frac{\partial u_{\sigma}}{\partial s}(s, x)=-\int_{M} H(s, x, y) \mathcal{L}_{y} \eta\left(\frac{d(x, y)}{\sigma}\right) d y
$$

and this tends to zero as $\sigma \rightarrow \infty$. The result is that $u(x)=\int_{M} H(s, x, y) d y$ is independent of $s$. We have

$$
u(x)-u_{\sigma}(s, x)=\int_{M} H(s, x, y)(1-\eta)\left(\frac{d(x, y)}{\sigma}\right) d y .
$$

For $\sigma>0$ fixed, the integrand is supported where $2 d(x, y) \geq \sigma$. Since

$$
H(s, x, y) \lesssim s V(x, y)^{-1} d(x, y)^{-2} \quad \text { for } \quad s \leq d(x, y)^{2}
$$

it follows from Remark 2.1.1 and the dominated convergence theorem that $u_{\sigma}(s, x) \rightarrow u(x)$ as $s \rightarrow 0$. The same estimate

$$
H(s, x, y) \lesssim s V(x, y)^{-1} d(x, y)^{-2}
$$

shows that for any $\epsilon>0$ and any $x \in M$ there is a constant $A>1$ so that

$$
\int_{d(x, y) \geq A s^{1 / 2}} H(s, x, y) d y \leq \epsilon \int_{M} H(s, x, y) d y=\epsilon u(x) .
$$

A standard argument then shows that if $f$ is bounded on $M$ and continuous at $x \in M$ then

$$
\lim _{s \rightarrow 0} \int_{M} H(s, x, y) f(y) d y=u(x) f(x) .
$$

If we apply this to $f(y)=\eta\left(\sigma^{-1} d(x, y)\right)$, we see that $u(x)=\eta(0)=1$. Thus $u(s, x) \equiv 1$, proving conclusion (4) of Proposition 2.3.1.

As is well-known, conclusion (6) of Proposition 2.3.1 follows from the facts that

$$
\int_{M}|H(s, x, y)| d y=\int_{M}|H(s, x, y)| d x=1
$$

which are themselves immediate consequences of (4) and (5). 
To establish conclusion (7), first note that the spectral theorem shows that $\lim _{s \rightarrow 0}|| H_{s}[\varphi]-\varphi \|_{L^{2}(M)}=0$. Next, since $\mathcal{L}_{x} H(s, x, y)=\mathcal{L}_{y} H(s, x, y)$, it follows that if $\varphi \in \mathcal{C}_{0}^{\infty}(M)$, then for any integer $N, \mathcal{L}^{N} H_{s}[\varphi]=H_{s}\left[\mathcal{L}^{n} \varphi\right]$. Thus for any $N, \lim _{s \rightarrow 0}\left\|\mathcal{L}^{n} H_{s}[\varphi]-\mathcal{L}^{n} \varphi\right\|_{L^{2}(M)}=\left\|H_{s}\left[\mathcal{L}^{n} \varphi\right]-\mathcal{L}^{n} \varphi\right\|_{L^{2}(M)}=$ 0 . Since $\mathcal{L}$ satisfies a subelliptic estimate, we obtain convergence of $H_{s}[\varphi]$ to $\varphi$ in any Sobolev space. Proposition 2.3.1 is therefore proved.

We shall next look at the behavior of $H_{s}[f]$ as $s \rightarrow \infty$. To do this we consider the spectral resolution of the operators $H_{s}=e^{-s \mathcal{L}}$ given by

$$
H_{s}=\int_{0}^{\infty} e^{-\mu s} d E(\mu)
$$

Here $E(\mu)$ is an increasing family of orthogonal projections, for $0 \leq \mu<\infty$, with $E(\mu) \rightarrow I$, as $\mu \rightarrow \infty$; we set $E_{0}=E(0)$. $E_{0}$ is just the orthogonal projection of $L^{2}(M)$ onto the null space of the (closed) operator $\mathcal{L}$.

Proposition 2.3.3 Let $f \in L^{2}(M)$. In case (A) when $M$ is compact, we have

$$
E_{0}(f)=\frac{1}{|M|} \int_{M} f d x
$$

There exists a $\mu_{1}>0$, so that

$$
\left\|H_{s}(f)-E_{0}(f)\right\|_{L^{2}} \leq e^{-\mu_{1} s}\|f\|_{L^{2}} .
$$

In case $(B)$ when $M$ is non-compact, we have $E_{0}[f] \equiv 0$, and

$$
\left\|H_{s}(f)\right\|_{L^{2}} \rightarrow 0, \text { as } s \rightarrow \infty \text {. }
$$

Proof. Consider the compact case first. If $E_{0}\left(f_{0}\right)=f_{0}$, then $H_{s}\left(f_{0}\right)=f_{0}$, for all $s>0$; however $u(s, x)=H_{s}\left(f_{0}\right)(x)$ satisfies the heat equation

$$
\frac{\partial u}{\partial s}+\mathcal{L}_{x}(u(s, x))=0
$$

therefore $f_{0} \in C^{\infty}$, and $\mathcal{L}\left(f_{0}\right)=0$. The compactness of $M$ allows us to integrate by parts, and so

$$
0=\left(\mathcal{L} f_{0}, f_{0}\right)=\sum_{j=1}^{k}\left(X_{j} f_{0}, X_{j} f_{0}\right),
$$

which implies $X_{j} f_{0}=0,1 \leq j \leq k$. As a result, $f_{0}$ is constant. Thus the subspace corresponding to $E_{0}$ consists of the constant functions, and

$$
E_{0}(f)=\frac{1}{|M|} \int_{M} f d x
$$


Next, consider the resolvent $R=R(1, \mathcal{L})=(I+\mathcal{L})^{-1}$. In view of kernel estimate 2.12 and the compactness of $M$, it is clear that $R$ is a compact operator on $L^{2}(M)$. Hence the eigenvalues of $R$ are discrete and accumulate only at 0 ; as a result the spectrum of $\mathcal{L}$ is discrete and its eigenvalues accumulate only at infinity. Thus $\mathcal{L}$ has a smallest strictly positive eigenvalue $\mu_{1}$. The fact that

$$
H_{s}=\int_{0}^{\infty} e^{-s \mu} d E(\mu)
$$

then establishes (2.14).

Turning to the non-compact case (B), we observe that

$$
\left|H_{s}(f)(x)\right| \leq\left(\int_{M}|H(s, x, y)|^{2} d y\right)^{1 / 2}\|f\|_{L^{2}} .
$$

However

$$
\int_{M}|H(s, x, y)|^{2} d y \lesssim V_{\sqrt{s}}(x)^{-2} \int_{d(x, y) \leq \sqrt{s}} d y+\int_{d(x, y) \geq \sqrt{s}} \frac{d y}{V(x, y) d(x, y)^{3}}
$$

by the estimates in Proposition 2.3.1, since $V(x, y) \geq c d(x, y)^{3}$. Therefore by (2.8) we have that

$$
\int|H(s, x, y)|^{2} d y \rightarrow 0, \quad \text { as } s \rightarrow \infty .
$$

So if $H_{s}\left(f_{0}\right)=f_{0}$, it follows that $f_{0}=0$, and hence $E_{0} \equiv 0$. It follows therefore that in this case,

$$
\left\|H_{s}(f)\right\|^{2}=\int_{0^{+}}^{\infty} e^{-2 \mu s} d(E(\mu) f, f) \rightarrow 0, \quad \text { as } s \rightarrow \infty
$$

and Proposition 2.3.3 follows by the dominated convergence theorem. This completes the proof of Proposition 2.3.3.

We record here related conclusions that are valid in both cases (A) and (B). If $f \in L^{2}(M)$ then

$$
\lim _{s \rightarrow 0}\left\|s \frac{\partial H_{s}}{\partial s}[f]\right\|_{L^{2}}=\lim _{s \rightarrow \infty}\left\|s \frac{\partial H_{s}}{\partial s}[f]\right\|_{L^{2}}=0 .
$$

In fact,

$$
\left\|s \frac{\partial H_{s}}{\partial s}[f]\right\|_{L^{2}}^{2}=\int_{0^{+}}^{\infty}(s \mu)^{2} e^{-2 s \mu} d(E(\mu)[f], f)
$$

and this tends to zero if either $s \rightarrow 0$ or $s \rightarrow \infty$, by the dominated convergence theorem. 


\subsection{A reproducing identity}

Define a bounded operator $Q_{s}=2 s \frac{\partial H_{s}}{\partial s}, s>0$ on $L^{2}(M)$ and let $Q_{s}^{2}=Q_{s} \cdot Q_{s}$.

Proposition 2.4.1 For $f \in L^{2}(M)$,

$$
\int_{0}^{\infty} Q_{s}^{2}[f] \frac{d s}{s}=f-E_{0}[f], \quad f \in L^{2}(M)
$$

where the integral on the left is defined as $\lim _{\epsilon \rightarrow 0} \int_{\epsilon}^{1 / \epsilon} Q_{s}^{2}[f] \frac{d s}{s}$, with the limit taken in the $L^{2}$ norm.

Proof. Since $H_{s}=e^{-s \mathcal{L}}$, it follows that $Q_{s}^{2}=4 s^{2} \mathcal{L}^{2} e^{-2 s \mathcal{L}}=s^{2} \frac{\partial^{2}}{\partial s^{2}}\left(H_{2 s}\right)$. Therefore

$$
\begin{aligned}
\int_{\epsilon}^{1 / \epsilon} Q_{s}^{2}[f] \frac{d s}{s} & =\int_{\epsilon}^{1 / \epsilon} s \frac{\partial^{2}}{\partial s^{2}}\left(H_{2 s}[f]\right) d s \\
& =\int_{\epsilon}^{1 / \epsilon} \frac{\partial}{\partial s}\left(s \frac{\partial}{\partial s} H_{2 s}(f)\right) d s-\int_{\epsilon}^{1 / \epsilon} \frac{\partial}{\partial s} H_{2 s} d s
\end{aligned}
$$

On the last line, one term goes to zero as $\epsilon \rightarrow 0$, in view of (2.16); the other terms goes to $f-E_{0}(f)$ in view of Proposition 2.3.3. Thus (2.17) is established.

\subsection{The square function}

For $f \in L^{2}(M)$ we define the square function $S(f)$ by

$$
(S[f](x))^{2}=\int_{0}^{\infty}\left|Q_{s}[f](x)\right|^{2} \frac{d s}{s}
$$

\section{Proposition 2.5.1}

1. For $f \in L^{2}(M)$,

$$
\|S[f]\|_{L^{2}(M)}^{2}+\left\|E_{0}(f)\right\|_{L^{2}(M)}^{2}=\|f\|_{L^{2}(M)}^{2} .
$$

2. For $1<p<\infty$, if $f \in L^{p}(M)$ then

$$
\|S[f]\|_{L^{p}(M)}+\left\|E_{0}(f)\right\|_{L^{p}(M)} \approx\|f\|_{L^{p}(M)} .
$$

The $L^{2}$ equality (1) follows from the identity of Proposition 2.4.1 by taking the inner product with $f$. The heart of the matter is the $L^{p}$ estimates (2). To see these, one applies the abstract Littlewood-Paley theory in [Ste70]. In fact, Proposition 2.3.1 shows that $H_{s}, 0<s<\infty$, is a symmetric diffusion semi-group in the sense of that reference, and the results in Chapter 4 of [Ste70] then establish the proposition. 


\subsection{The product case}

We now assume that $\widetilde{M}=M_{1} \times M_{2}$ where each $M_{i}$ is as above. For each $i$, we have a heat operator $H_{s_{i}}^{i}$, and a corresponding $Q_{s_{i}}^{i}$, together with the projection $E_{0}^{i}$. If $f$ is a function on $\widetilde{M}$ we define $Q_{s_{1}}^{1} \cdot Q_{s_{2}}^{2}=Q_{s_{1}}^{1} \otimes Q_{s_{2}}^{2}$, with $Q^{1}$ acting on the $M_{1}$ variable, and $Q^{2}$ acting on the $M_{2}$ variable. The product square function is then $\widetilde{S}$ given by

$$
(\widetilde{S}(f)(x))^{2}=\int_{0}^{\infty} \int_{0}^{\infty}\left|Q_{s_{1}}^{1} \cdot Q_{s_{2}}^{2}(f)(x)\right|^{2} \frac{d s_{1} d s_{2}}{s_{1} s_{2}}
$$

Proposition 2.6.1 For $1<p<\infty$,

$$
\|\widetilde{S}(f)\|_{L^{p}(\widetilde{M})}+\left\|E_{0}^{1}(f)\right\|_{L^{p}(\widetilde{M})}+\left\|E_{0}^{2}(f)\right\|_{L^{p}(\widetilde{M})} \approx\|f\|_{L^{p}(\widetilde{M})},
$$

This can be derived from Proposition 2.5.1 if we observe that this holds as well for functions which take their values in a Hilbert space.

To begin with, suppose $E_{0}^{1}(f)=E_{0}^{2}(f)=0$. For each fixed $x_{2} \in M_{2}$, set $F_{x_{2}}\left(x_{1}\right)=Q_{s_{2}}^{2}(f)\left(x_{1}, x_{2}\right)$, and consider it as a function on $M_{1}$, with values in the Hilbert space $L^{2}\left(\frac{d s_{2}}{s_{2}}\right)$. Note that $E_{0}^{1}\left(F_{x_{2}}\right)=0$. Therefore, by Proposition 2.5.1,

$$
\begin{aligned}
\left\|\widetilde{S}[f]\left(\cdot, x_{2}\right)\right\|_{L^{p}\left(M_{1}\right)} & =\left\|\left(\iint\left|Q_{s_{1}}^{1} \cdot Q_{s_{2}}^{2}[f]\left(\cdot, x_{2}\right)\right|^{2} \frac{d s_{1} d s_{2}}{s_{1} \cdot s_{2}}\right)^{1 / 2}\right\|_{L^{p}\left(M_{1}\right)} \\
& \approx\left\|F_{x_{2}}(\cdot)\right\|_{L^{p}\left(M_{1}\right)} \\
& =\left\|\left(\int\left|Q_{s_{2}}^{2}[f]\left(\cdot, x_{2}\right)\right|^{2} \frac{d s_{2}}{s_{2}}\right)^{1 / 2}\right\|_{L^{p}\left(M_{1}\right)} \\
& \approx\left\|f\left(\cdot, x_{2}\right)\right\|_{L^{p}\left(M_{1}\right)} .
\end{aligned}
$$

Now raise both sides to the $p^{\text {th }}$ power and integrate in $x_{2} \in M_{2}$ to obtain (2.19) in this case.

For the general case write $f=f_{0}+f_{1}+f_{2}+f_{3}$ where

$$
f_{0}=\left(1-E_{0}^{1}\right)\left(1-E_{0}^{2}\right)[f], f_{1}=E_{0}^{1}[f], f_{2}=E_{0}^{2}[f], \text { and } f_{3}=-E_{0}^{1} E_{0}^{2}[f] .
$$

Note that

$$
Q_{s_{1}}^{1} \cdot Q_{s_{2}}^{2}\left[f_{0}\right]=Q_{s_{1}}^{1} \cdot Q_{s_{2}}^{2}[f]
$$

while

$$
\left\|E_{0}^{1} E_{0}^{2}[f]\right\|_{L^{p}(\widetilde{M})} \leq\left\|E_{0}^{2}[f]\right\|_{L^{p}(\widetilde{M})}
$$

by Hölder's inequality. 


\section{3. $L^{p}$ estimates; the single factor case}

In this section we again consider the special case where $M=M_{i}$ for some $i$, and we suppose that $\left\{X_{1}, \ldots, X_{k}\right\}$ are smooth vector fields on $M$, which together with their commutators span the tangent space at every point. We study $L^{p}$-boundedness of a class of singular integral operators on $M$ determined by the geometry studied in section 2.1 .

Our operators will be characterized in part in terms of their action on bump functions. Loosely speaking, these are defined as follows: we say $\varphi$ is a bump function associated to a ball $B\left(x_{0}, \delta\right)$, if it is supported in that ball, and satisfies the differential inequalities $\left|\partial_{X}^{a} \varphi\right| \lesssim \delta^{-a}$, for all monomials $\partial_{X}$ in $X_{1}, \ldots, X_{k}$ of degree $a$, and all $a \geq 0$. A useful way to construct such $\varphi$ is by setting $\varphi(x)=\eta\left(\frac{d(x, y)}{\delta}\right)$ with $\eta$ a fixed $C^{\infty}$ function on $(0, \infty), \eta(u)=0$, when $u \geq 1$. Here $d(x, y)$ is the regularized distance used in Section 2 . We give precise definitions in the next subsection.

\subsection{A class of singular integral operators on $M$}

We consider a class of singular integral operators $T$ which we assume initially are given as mappings from $C_{0}^{\infty}(M)$ to $C^{\infty}(M)$. We suppose the operator $T$ has a distribution kernel $K(x, y)$ which is $C^{\infty}$ away from the diagonal of $M \times M$, and we suppose the following four properties hold:

(I-1) If $\varphi, \psi \in \mathcal{C}_{0}^{\infty}(M)$ have disjoint supports, then

$$
\langle T \varphi, \psi\rangle=\int_{M \times M} K(x, y) \varphi(y) \psi(x) d x d y .
$$

(I-2) If $\varphi$ is a normalized bump function associated to a ball of radius $r$, then $\left|\partial_{X}^{a} T \varphi\right| \lesssim r^{-a}$. More precisely, for each integer $a \geq 0$, there is another integer $b \geq 0$ and a constant $M_{a, b}$ so that whenever $\varphi$ is a $C^{\infty}$ function supported in a ball $B\left(x_{0}, r\right)$, then

$$
\sup _{x \in M} r^{a}\left|\left(\partial_{X}^{a} T \varphi\right)(x)\right| \leq M_{a, b} \sup _{c \leq b} \sup _{x \in B\left(x_{0}, r\right)} r^{c}\left|\partial_{X}^{c}(\varphi)\right| .
$$

(I-3) If $x \neq y$, then for every $a \geq 0$

$$
\left|\partial_{X, Y}^{a} K(x, y)\right| \lesssim d(x, y)^{-a} V(x, y)^{-1}
$$

(I-4) Properties (I-1) through (I-3) also hold with $x$ and $y$ interchanged. That is, these properties also hold for the adjoint operator $T^{t}$ defined by

$$
\left\langle T^{t} \varphi, \psi\right\rangle=\langle T \psi, \varphi\rangle .
$$


Our main result in this section is as follows:

Theorem 3.1.1 Each singular integral operator $T$ satisfying the conditions (I-1) through (I-4) extends to a bounded operator on $L^{p}(M)$ whenever $1<p<\infty$.

\subsection{The relationship with NIS operators}

We want to explain the relationship between the class of operators defined in subsection 3.1 by properties (I-1) through (I-4), and the notion of NIS operators smoothing of order zero. A class of NIS operators smoothing of order $m$ was introduced in [NRSW89] and [CNS92]. When $m=0$, this class is closely connected to but more restrictive than the operators considered in subsection 3.1. For an operator $T$, in addition to the properties (I-1) through (I-4), it is assumed that there is a family of operators $T_{\epsilon}[f](x)=\int_{M} K_{\epsilon}(x, y) f(y) d y$ where $K_{\epsilon} \in \mathcal{C}^{\infty}(M \times M)$ which satisfies properties (I-2) through (I-4) uniformly in $\epsilon$ such that $T_{\epsilon}[f] \rightarrow T[f]$ in $\mathcal{C}^{\infty}$. This condition was imposed in order to prove that the operators in question formed an algebra under composition. However, as pointed out in [Koe02], it was implicitly assumed that the identity operator is an NIS operator smoothing of order zero. In order to overcome this difficulty, a modification of the definition of NIS operators is given in [Koe02] where an a priori Sobolev inequality is imposed for the operator $T$ instead of the existence of an approximating family $T_{\epsilon}$. This definition was used in [NS01a].

In our present context, we do not need to establish that operators satisfying properties (I-1) through (I-4) form an algebra. Thus both previously defined classes of NIS operators smoothing of order zero are covered by Theorem 3.1.1.

Remark 3.2.1 Conclusions (1), (2), (3), (4) and (7) of Proposition 1.3.1 now show that the identity operator on $L^{2}(M)$ is an NIS operator smoothing of order zero in the sense of [NRSW89] and [CNS92].

\subsection{Singular integrals and square functions}

In addition to the use of the Littlewood-Paley theory in Section 2, the key tool in proving Theorem 3.1.1 is the following estimate:

Proposition 3.3.1 For each operator $T$ satisfying the conditions (I-1) through (I-4),

$$
\left|Q_{t} T Q_{s} f(x)\right| \lesssim(t / s)^{1 / 2} \mathcal{M}(f)(x), \text { if } t \leq s
$$

where $\mathcal{M}$ is the standard maximal function. A similar result holds if $s \leq t$. 
We shall prove this proposition by writing

$$
Q_{s}=\sum_{k=0}^{\infty} Q_{s}^{k}, \quad Q_{t}=\sum_{j=0}^{\infty}\left(Q_{t}^{j}\right)^{*}
$$

and showing:

$$
\left|\left(Q_{t}^{j}\right)^{*} T Q_{s}^{k} f(x)\right| \lesssim 2^{-2 k} \cdot 2^{-2 j} \min \left\{\frac{2^{j} t^{1 / 2}}{2^{k} s^{1 / 2}}, \frac{2^{k} s^{1 / 2}}{2^{j} t^{1 / 2}}\right\} \mathcal{M} f(x) .
$$

Here $Q_{s}^{k}$ will be like $Q_{s}$, except that its support is restricted to $d(x, y) \lesssim$ $2^{k} \sqrt{s}$. Details of this decomposition will be given in section 3.5 below. Note that the second decomposition for $Q_{t}$ follows from the first, because $Q_{t}^{*}=Q_{t}$.

\subsection{Two observations}

The proof of Proposition 3.3.1 is based on the following observations.

Observation 3.4.1 Suppose that $\varphi_{\rho}\left(x, x_{0}\right)$ is a bump-function (in $x$ ), associated to $B\left(x_{0}, \rho\right)$ and that $\psi_{r}\left(y, y_{0}\right)$ is a bump-function (in $y$ ) associated to $B\left(y_{0}, r\right)$. Assume further that

$$
\int \varphi_{\rho}\left(x, x_{0}\right) d x=\int \psi_{r}\left(y, y_{0}\right) d y=0 .
$$

Let $K(x, y)$ be the distribution kernel of an operator $T$ satisfying $(I-1)$ to (I-4), and set

$$
I=\iint K(x, y) \varphi_{\rho}\left(x, x_{0}\right) \psi_{r}\left(y, y_{0}\right) d x d y
$$

If $r \leq \rho$ then

$$
|I| \lesssim \frac{r}{\rho} \cdot V_{r}\left(y_{0}\right)
$$

$$
|I| \lesssim \frac{r}{d\left(x_{0}, y_{0}\right)} \cdot \frac{V_{\rho}\left(x_{0}\right) \cdot V_{r}\left(y_{0}\right)}{V\left(x_{0}, y_{0}\right)} \quad \text { if } d\left(x_{0}, y_{0}\right) \geq 10 \rho
$$

To see this, first consider (a). Note that since $\varphi_{\rho}$ is a bump-function associated to a ball of radius $\rho$, conditions (I-2) and (I-4) show that

$$
\Phi(y)=\int K(x, y) \varphi_{\rho}\left(x, x_{0}\right) d x
$$

satisfies $\left|\partial_{Y}^{a} \Phi(y)\right| \lesssim \rho^{-a}$. But

$$
I=\int \Phi(y) \psi_{r}\left(y, y_{0}\right) d y=\int\left[\Phi(y)-\Phi\left(y_{0}\right)\right] \psi_{r}\left(y, y_{0}\right) d y
$$


Let $y \in B\left(y_{0}, r\right)$, and let $\gamma(t)$ be an appropriate path joining $y_{0}$ to $y$ whose tangent is in the span of $\left\{X_{1}, \ldots, X_{k}\right\}$. Then $\left|\Phi(y)-\Phi\left(y_{0}\right)\right| \lesssim \rho^{-1} r$ since

$$
\Phi(y)-\Phi\left(y_{0}\right)=\int_{0}^{r} \frac{\partial}{\partial t}(\Phi(\gamma(t))) d t
$$

and hence

$$
\left|\Phi(y)-\Phi\left(y_{0}\right)\right| \lesssim r \sum_{j=1}^{k} \sup \left|X_{j}(\Phi)\right| \lesssim \frac{r}{\rho}
$$

Since

$$
\int\left|\psi_{r}\left(y, y_{0}\right)\right| d y \lesssim V_{r}\left(y_{0}\right)
$$

this establishes assertion (a).

To prove assertion (b) write

$$
I=\iint\left[K(x, y)-K\left(x, y_{0}\right)\right] \varphi_{\rho}\left(x, x_{0}\right) \psi_{r}\left(y, y_{0}\right) d x d y .
$$

If $x \in B\left(x_{0}, \rho\right), y \in B\left(y_{0}, r\right), d\left(x_{0}, y_{0}\right) \geq 10 \rho$ and $\rho \geq r$ then

$$
\left|K(x, y)-K\left(x, y_{0}\right)\right| \lesssim \frac{r}{V\left(x_{0}, y_{0}\right) \cdot d\left(x_{0}, y_{0}\right)} .
$$

This fact is a consequence of (I-3) and the argument leading to (3.1). Since

$$
\iint\left|\varphi_{\rho}\left(x, x_{0}\right) \psi_{r}\left(y, y_{0}\right)\right| d x d y \lesssim V_{\rho}\left(x_{0}\right) V_{r}\left(y_{0}\right),
$$

this establishes assertion (b). (Clearly an analogous result holds if $r \geq \rho$.) This completes the discussion of Observation 3.4.1.

We next replace $\varphi_{\rho}$ and $\psi_{r}$ by adjusted bump-functions. That is, define

$$
\begin{aligned}
\varphi_{\rho}^{*}\left(x, x_{0}\right) & =\frac{1}{V_{\rho}\left(x_{0}\right)} \varphi_{\rho}\left(x, x_{0}\right) \\
\psi_{r}^{*}\left(y, y_{0}\right) & =\frac{1}{V_{r}\left(y_{0}\right)} \psi_{r}\left(y, y_{0}\right)
\end{aligned}
$$

and set

$$
I^{*}=\int K(x, y) \varphi_{\rho}^{*}\left(x, x_{0}\right) \psi_{r}^{*}\left(y, y_{0}\right) d x d y .
$$

The following is now an immediate consequence of Observation 3.4.1.

Observation 3.4.2 For the adjusted integral $I^{*}$ we have

(a) $\left|I^{*}\right| \lesssim \frac{r}{\rho} \frac{1}{V_{\rho}\left(x_{0}\right)}$

(b) $\quad\left|I^{*}\right| \lesssim \frac{r}{d\left(x_{0}, y_{0}\right) V\left(x_{0}, y_{0}\right)}$, if $d\left(x_{0}, y_{0}\right) \geq 10 \rho$. 


\subsection{Decomposition of $Q_{s}$}

Let $q_{s}(x, y)$ be the kernel of $Q_{s}=2 s \frac{\partial H_{s}}{\partial s}$. Note that

$$
\int q_{s}(x, y) d x=0
$$

since $Q_{s}(1)=0$, in view of conclusion (5) of Proposition 2.3.1.

Lemma 3.5.1 We can write

$$
q_{s}(x, y)=\sum_{k=0}^{\infty} 2^{-2 k} \varphi_{2^{k} \sqrt{s}}^{*}(x, y)
$$

where $\varphi_{2^{k} \sqrt{s}}^{*}(x, y)$ is an adjusted bump function (in $x$ ) for the ball of radius $\sqrt{s} 2^{k}$ centered at $y$. Moreover for each $k$

$$
\int \varphi_{2^{k} \sqrt{s}}^{*}(x, y) d x=0
$$

Similarly we can write

$$
q_{t}(x, y)=\sum_{j=0}^{\infty} 2^{-2 j} \psi_{2^{j} \sqrt{t}}^{*}(x, y)
$$

where $\psi_{\sqrt{t} 2^{j}}^{*}(x, y)$ is a normalized bump function (in $y$ ) for the ball of radius $\sqrt{t} 2^{j}$ centered at $x$. Moreover for each $j$

$$
\int \psi_{2^{j} \sqrt{t}}^{*}(x, y) d y=0
$$

Remark 3.5.2 The factor $2^{-2 k}$ can be replaced by $2^{-k N}$, for any $N>0$. Similarly the factor $2^{-2 j}$ can be replaced by $2^{-j N}$.

Proof of Lemma 3.5.1 Fix a function $\alpha \in \mathcal{C}^{\infty}(\mathbb{R})$ so that $\alpha(t)=1$ if $t \leq 1 / 2$ and $\alpha(t)=0$ if $t \geq 1$. Let

$$
\begin{aligned}
& A_{0}(x, y)=\alpha\left(\frac{d(x, y)}{\sqrt{s}}\right) q_{s}(x, y), \\
& A_{k}(x, y)=\left[\alpha\left(\frac{d(x, y)}{2^{k} \sqrt{s}}\right)-\alpha\left(\frac{d(x, y)}{2^{k-1} \sqrt{s}}\right)\right] q_{s}(x, y), \quad k \geq 1 .
\end{aligned}
$$

Then

$$
q_{s}(x, y)=\sum_{k=0}^{\infty} A_{k}(x, y)
$$


Since $Q_{t}=2 t \frac{\partial H}{\partial t}$, it follows from the estimates of the heat kernel and its derivatives in Proposition 2.3.1 that for all $N \geq 0$

$$
\left|q_{s}(x, y)\right| \lesssim \frac{1}{V_{\sqrt{s}}(y)}\left(\frac{s}{s+d^{2}(x, y)}\right)^{N}
$$

As a result it is easy to see that for all $N^{\prime} \geq 0$

$$
\left|A_{k}(x, y)\right| \lesssim 2^{-k N^{\prime}} \frac{1}{V_{2^{k} \sqrt{s}}(y)} .
$$

Note that if we define $a_{k}$ by

$$
a_{k}(y)=\int A_{k}(x, y) d x
$$

then $a_{k}(y)=O\left(2^{-k N^{\prime}}\right)$. Moreover,

$$
\sum_{k=0}^{\infty} a_{k}(y)=\int q_{s}(x, y) d x=0
$$

since $Q_{s}[1]=0$. Put $s_{k}=\sum_{j \leq k} a_{j}$, and note that $s_{k}=-\sum_{j>k} a_{j}$ since $\sum_{j=0}^{\infty} a_{j}=0$. It follows that $s_{k}=O\left(2^{-k N^{\prime}}\right)$.

Next, let

$$
\tilde{\eta}_{k}(x)=\tilde{\eta}_{k}(x, y)=\alpha\left(\frac{d(x, y)}{2^{k} \sqrt{s}}\right) .
$$

Then $\tilde{\eta}_{k}$ is a bump function for the ball $B\left(y, 2^{k} \sqrt{s}\right)$. Set

$$
\eta_{k}(x)=\tilde{\eta}_{k}(x)\left[\int \tilde{\eta}_{k}(x) d x\right]^{-1}
$$

so that $\eta_{k}$ is an "adjusted" bump function for that ball. Set

$$
\tilde{A}_{k}(x, y)=A_{k}(x, y)-a_{k}(y) \eta_{k}(x)+s_{k}(y)\left(\eta_{k}-\eta_{k+1}\right)(x) .
$$

Then

$$
\sum_{k=0}^{\infty} \tilde{A}_{k}(x, y)=\sum_{k=0}^{\infty} A_{k}(x, y)-\sum_{k=0}^{\infty} a_{k}(y) \eta_{k}(x)+\sum_{k=0}^{\infty} s_{k}(y)\left(\eta_{k}-\eta_{k+1}\right)(x) .
$$

However, $\sum_{k} A_{k}=q_{s}$ and $\sum_{k} a_{k} \eta_{k}=\sum_{k} s_{k}\left(\eta_{k}-\eta_{k+1}\right)$. Also, observe that $\int \tilde{A}_{k}(x, y) d x=0$, all $k$. Thus, if we take

$$
\varphi_{2^{k} \sqrt{s}}^{*}(x, y)=2^{2 k} \tilde{A}_{k}(x, y),
$$

Lemma 3.5.1 is proved. 
We turn now to the proof of Proposition 3.3.1. Let $Q_{s}^{k}$ be the operator whose kernel is $2^{-2 k} \varphi_{2^{k} \sqrt{s}}^{*}(x, y)$ and let $\left(Q_{t}^{j}\right)^{*}$ be the operator with the adjoint kernel $2^{-2 j} \psi_{2^{j} \sqrt{t}}^{*}(y, x)$. Apply Observation 2 to $\left(Q_{t}^{j}\right)^{*} T Q_{s}^{k}$. The result is easily seen to imply that

$$
\left|\left(Q_{t}^{j}\right)^{*} T Q_{s}^{k} f(x)\right| \lesssim 2^{-2 k} \cdot 2^{-2 j} \min \left\{\frac{2^{j} t^{1 / 2}}{2^{k} s^{1 / 2}}, \frac{2^{k} s^{1 / 2}}{2^{j} t^{1 / 2}}\right\} \mathcal{M}(f)(x)
$$

This follows directly from the two inequalities in Observation 2, together with the estimate (2.9) for the maximal function, with $a=1$.

However,

$$
\left|Q_{t} T Q_{s} f(x)\right| \leq \sum_{k, j \geq 0}\left|\left(Q_{t}^{j}\right)^{*} T Q_{s}^{k} f(x)\right|
$$

Since

$$
\sum_{k \geq 0} \sum_{j \geq 0} 2^{-2 k} 2^{-2 j} \min \left\{\frac{2^{j} t^{1 / 2}}{2^{k} s^{1 / 2}}, \frac{2^{k} s^{1 / 2}}{2^{j} t^{1 / 2}}\right\} \lesssim \min \left(\frac{t^{1 / 2}}{s^{1 / 2}}, \frac{s^{1 / 2}}{t^{1 / 2}}\right)
$$

the statement of Proposition 3.3.1 is established.

\subsection{Proof of Theorem 3.1.1}

Consider first the case (B) of the "model domain", when $M$ is non-compact and $E=0$ in Proposition 2.5.1 We have by (2.17),

$$
Q_{t} T=Q_{t} T \int_{0}^{\infty} Q_{s} \cdot Q_{s} \frac{d s}{s}=\int_{0}^{\infty} Q_{t} T Q_{s} \cdot Q_{s} \frac{d s}{s}
$$

Hence

$$
\left|Q_{t} T(f)\right| \lesssim \int_{0}^{\infty} \min \left(\frac{t^{1 / 2}}{s^{1 / 2}}, \frac{s^{1 / 2}}{t^{1 / 2}}\right) \mathcal{M}\left(Q_{s} f\right) \frac{d s}{s}
$$

Now by Hardy's inequality if

$$
F(t)=\int_{0}^{\infty} \min \left(\frac{t^{1 / 2}}{s^{1 / 2}}, \frac{s^{1 / 2}}{t^{1 / 2}}\right) G(s) \frac{d s}{s},
$$

then

$$
\int_{0}^{\infty}|F(t)|^{2} \frac{d t}{t} \leq C \int_{0}^{\infty}|G(s)|^{2} \frac{d s}{s}
$$

Hence

$$
\int_{0}^{\infty}\left|Q_{t}(T f)\right|^{2} \frac{d t}{t} \leq C \int_{0}^{\infty} \mathcal{M}\left(Q_{s} f\right)^{2} \frac{d s}{s}
$$

and the result is obtained by appealing to Proposition 2.5.1 and the vectorvalued maximal inequality $(2.11)$, with $f_{s}=s^{-\frac{1}{2}} Q_{s}[f]$. 
In the compact case, (situation $(\mathrm{A})$ ), we have the additional term $E_{0}(T f)$. Its estimate is easy because $E_{0}(F)=c(F, 1)$, with $c=|M|^{-1}$, and therefore $E_{0}(T f)=c(T f, 1)=c\left(f, T^{*}(1)\right)$. However, according to our hypotheses $T^{*}(1)$ is a $C^{\infty}$ function on $M$, and hence obviously $\left\|E_{0} T(f)\right\|_{L^{p}} \leq A\|f\|_{L^{p}}$ for all $p, 1 \leq p \leq \infty$, and the proof of Theorem 3.1.1 is complete.

\section{The product case: two factors}

We now consider $\widetilde{M}=M_{1} \times M_{2}$, where each $M_{i}$ is as above, and now define an appropriate notion of product singular integrals.

\subsection{A class of singular integrals}

We shall consider linear mappings $T$, initially defined from $C_{0}^{\infty}$ to $C^{\infty}(\widetilde{M})$, which have an associated distribution kernel $K(x, y)$, which are $C^{\infty}$ away from the "cross" $=\left\{(x, y): x_{1}=y_{1}\right.$, or $\left.x_{2}=y_{2} ; x=\left(x_{1}, x_{2}\right), y=\left(y_{1}, y_{2}\right)\right\}$ and which satisfy the following additional properties.

$$
\begin{aligned}
& \left\langle T\left(\varphi_{1} \otimes \varphi_{2}\right), \psi_{1} \otimes \psi_{2}\right\rangle= \\
& \quad=\int K\left(x_{1}, y_{1}, x_{2}, y_{2}\right) \varphi_{1}\left(y_{1}\right) \varphi_{2}\left(y_{2}\right) \psi_{1}\left(x_{1}\right) \psi_{2}\left(x_{2}\right) d y d x \\
& \text { whenever }\left\{\begin{array}{l}
\varphi_{1}, \psi_{1} \in C_{0}^{\infty}\left(M_{1}\right) \text { and have disjoint support, } \\
\varphi_{2}, \psi_{2} \in C_{0}^{\infty}\left(M_{2}\right) \text { and have disjoint support. }
\end{array}\right.
\end{aligned}
$$

(II-2) For each bump function $\varphi_{2}$ on $M_{2}$, and each $x_{2} \in M_{2}$, there exists a singular integral $T^{\varphi_{2}, x_{2}}$ (of the one-factor type) on $M_{1}$, so that

$$
\left\langle T\left(\varphi_{1} \otimes \varphi_{2}\right), \psi_{1} \otimes \psi_{2}\right\rangle=\int_{M_{2}}\left\langle T^{\varphi_{2}, x_{2}} \varphi_{1}, \psi_{1}\right\rangle \psi_{2}\left(x_{2}\right) d x_{2}
$$

Moreover, $x_{2} \mapsto T^{\varphi_{2}, x_{2}}$ is smooth and uniform in the sense that $T^{\varphi_{2}, x_{2}}$, as well as $\rho_{2}^{L} \partial_{X_{2}}^{L}\left(T^{\varphi_{2}, x_{2}}\right)$ for each $L \geq 0$, satisfy the conditions (I-1) to (I-4) uniformly.

(II-3) If $\varphi_{j}$ is a bump function on a ball $B^{j}\left(r_{j}\right)$ in $M_{j}$, then

$$
\left|\partial_{X_{1}}^{a_{1}} \partial_{X_{2}}^{a_{2}} T\left(\varphi_{1} \otimes \varphi_{2}\right)\right| \lesssim r_{1}^{-a} r_{2}^{-a_{2}}
$$

In (II-2) and (II-3) both inequalities are taken in the sense of (I-2) whenever $\varphi_{2}$ is a bump function for $B_{\left(r_{2}\right)}^{2}$ (in $\left.M_{2}\right)$. 


$$
\left|\partial_{X_{1}, Y_{1}}^{a_{1}} \partial_{X_{2}, Y_{2}}^{a_{2}} K\left(x_{1}, y_{1} ; x_{2}, y_{2}\right)\right| \lesssim \frac{d_{1}\left(x_{1}, y_{1}\right)^{-a_{1}} d_{2}\left(x_{2}, y_{2}\right)^{-a_{2}}}{V_{1}\left(x_{1}, y_{1}\right) V_{2}\left(x_{2}, y_{2}\right)}
$$

(II-5) The same conditions hold when the index 1 and 2 are interchanged, that is if the roles of $M_{1}$ and $M_{2}$ are interchanged.

(II-6) The same properties are assumed to hold for the 3 "transposes" of $T$, i.e. those operators which arise by interchanging $x_{1}$ and $y_{1}$, or interchanging $x_{2}$ and $y_{2}$, or doing both interchanges.

Remark 4.1.1 If $T_{j}$ are singular integral operators on $M_{j}$ (for the onefactor case), $j=1,2$, then $T=T_{1} \otimes T_{2}$ satisfies the above assumptions. Here $T^{\varphi_{2}, x_{2}}=T_{1}$ multiplied by the factor $T_{2}\left(\varphi_{2}\right)\left(x_{2}\right)$.

The main result of this section is the following:

Theorem 4.1.2 For $1<p<\infty$, each product singular integral satisfying conditions (II-1) to (II-6) extends to a bounded operator on $L^{p}\left(M_{1} \times M_{2}\right)$ to itself.

\subsection{Two additional observations}

To begin the proof of Theorem 4.1.2 we make two observation analogous to Observations 3.4.1 and 3.4.2 in Subsection 3.4.

\section{Observation 4.2.1 Suppose}

(i) $\varphi_{\rho}\left(y_{1}\right)$ is a bump function on $B_{\rho_{1}}^{1}\left(\bar{y}_{1}\right)$ (the ball of radius $\rho_{1}$, centered at $\left.\bar{y}_{1}\right)$

(ii) $\psi_{r_{1}}\left(x_{1}\right)$ is a bump function on $B_{r_{1}}^{1}\left(\bar{x}_{1}\right)$

(iii) $\varphi_{\rho_{2}}\left(y_{2}\right)$ is a bump function on $B_{\rho_{2}}^{2}\left(\bar{y}_{2}\right)$

(iv) $\psi_{r_{2}}\left(x_{2}\right)$ is a bump function on $B_{r_{2}}^{2}\left(\bar{x}_{2}\right)$.

Assume also

$$
\int \varphi_{\rho_{1}}\left(y_{1}\right) d y_{1}=\int \varphi_{\rho_{2}}\left(y_{2}\right) d y_{2}=\int \psi_{r_{1}}\left(x_{1}\right) d x_{1}=\int \psi_{r_{2}}\left(x_{1}\right) d x_{1}=0 .
$$

Let

$$
I=\left\langle T\left(\psi_{r_{1}} \otimes \psi_{r_{2}}\right), \varphi_{\rho_{1}} \otimes \varphi_{\rho_{2}}\right\rangle=\left\langle T^{t}\left(\varphi_{\rho_{1}} \otimes \varphi_{\rho_{2}}\right), \psi_{r_{1}} \otimes \psi_{r_{2}}\right\rangle .
$$

Suppose that $r_{1} \leq \rho_{1}, r_{2} \leq \rho_{2}$. Then we have the following estimates for $I$ : 
(a) For all values of the parameters,

$$
|I| \lesssim\left(\frac{r_{1}}{\rho_{1}}\right)\left(\frac{r_{2}}{\rho_{2}}\right) V_{r_{1}}^{1}\left(\bar{y}_{1}\right) V_{r_{2}}^{2}\left(\bar{y}_{2}\right)
$$

(b) If $d_{1}\left(\bar{x}_{1}, \bar{y}_{1}\right) \geq 10 \rho_{1}$,

$$
|I| \lesssim\left(\frac{r_{2}}{\rho_{2}}\right)\left(\frac{r_{1}}{d_{1}\left(\bar{x}_{1}, \bar{y}_{1}\right)}\right) \frac{V_{r_{2}}^{2}\left(\bar{y}_{2}\right) V_{\rho_{1}}^{2}\left(\bar{x}_{1}\right) V_{r_{1}}^{1}\left(\bar{y}_{1}\right)}{V^{1}\left(\bar{x}_{1}, \bar{y}_{1}\right)} ;
$$

(c) If $d_{1}\left(\bar{x}_{1}, \bar{y}_{1}\right) \geq 10 \rho_{1}$ and $d_{2}\left(\bar{x}_{2}, \bar{y}_{2}\right) \geq 10 \rho_{2}$,

$$
|I| \lesssim\left(\frac{r_{1}}{d_{1}\left(\bar{x}_{1}, \bar{y}_{1}\right)}\right)\left(\frac{r_{2}}{d_{2}\left(\bar{x}_{2}, \bar{y}_{2}\right)}\right) \frac{V_{\rho_{1}}^{1}\left(\bar{x}_{1}\right) V_{\rho_{2}}^{1}\left(\bar{x}_{2}\right) V_{r_{1}}^{2}\left(\bar{y}_{1}\right) V_{r_{2}}^{2}\left(\bar{y}_{2}\right)}{V^{1}\left(\bar{x}_{1}, \bar{y}_{1}\right) V^{2}\left(\bar{x}_{2}, \bar{y}_{2}\right)} .
$$

Similar results hold of the indices 1 and 2 are interchanged, as well as if the inequalities $r_{1} \leq \rho_{1}$ or $r_{2} \leq \rho_{2}$ are reversed.

Proof of (a):

Let

$$
\Phi\left(y_{1}, y_{2}\right)=T^{t}\left(\varphi_{\rho_{1}} \otimes \varphi_{\rho_{2}}\right)\left(y_{1}, y_{2}\right)
$$

Then

$$
I=\int \Phi\left(y_{1}, y_{2}\right) \psi_{r_{1}}\left(y_{1}\right) \psi_{r_{2}}\left(y_{2}\right) d y_{1} d y_{2}
$$

Since the integrals of the $\psi$ 's vanish we have

$$
I=\int\left[\Phi\left(y_{1}, y_{2}\right)-\Phi\left(y_{1}, \bar{y}_{2}\right)-\Phi\left(\bar{y}_{1}, y_{2}\right)+\Phi\left(\bar{y}_{1}, \bar{y}_{2}\right)\right] \psi_{r_{1}}\left(y_{1}\right) \psi_{r_{2}}\left(y_{2}\right) d y_{1} d y_{2}
$$

We need the following lemma, akin to the argument leading to (3.1):

Lemma 4.2.2 Suppose $\sup _{y_{1}, y_{2}}\left|D_{1} D_{2} \Phi\left(y_{1}, y_{2}\right)\right| \lesssim A$, where $D_{j}$ is any differentiation with respect to a distinguished vector field in $M_{j}$. Suppose $y_{1} \in B_{r_{1}}^{1}\left(\bar{y}_{1}\right)$ and $y_{2} \in B_{r_{2}}^{2}\left(\bar{y}_{2}\right)$. Then

$$
\left|\Phi\left(y_{1}, y_{2}\right)-\Phi\left(y_{1}, \bar{y}_{2}\right)-\Phi\left(\bar{y}_{1}, y_{2}\right)+\Phi\left(\bar{y}_{1}, \bar{y}_{2}\right)\right| \lesssim A r_{1} r_{2} .
$$

Proof. Since $y_{j} \in B_{r_{j}}^{j}\left(\bar{y}_{j}\right)$, there exists curves $t_{j} \rightarrow \gamma_{j}\left(t_{j}\right) \in \partial \Omega_{j}, 0 \leq t_{j} \leq$ $\bar{t}_{j}$, so that each $\dot{\gamma}_{j}\left(t_{j}\right)$ is a linear combination (with coefficients $\leq 1$ ) of good vector fields, and so that $\gamma_{j}(0)=y_{j}, \gamma_{j}\left(\bar{t}_{j}\right)=\bar{y}_{j}$, while $\bar{t}_{j} \lesssim r_{j}$. Then

$$
\begin{aligned}
\Phi\left(y_{1}, y_{2}\right)-\Phi\left(y_{1}, \bar{y}_{2}\right)-\Phi\left(\bar{y}_{1}, y_{2}\right) & +\Phi\left(\bar{y}_{1}, \bar{y}_{2}\right) \\
& =\iint_{R} \frac{\partial^{2}}{\partial t_{1} \partial t_{2}}\left[\Phi\left(\gamma_{1}\left(t_{1}\right), \gamma_{2}\left(t_{2}\right)\right)\right] d t, d t_{2}
\end{aligned}
$$

where $R$ is the rectangle $\left[0, \bar{t}_{1}\right] \times\left[0, \bar{t}_{2}\right]$. The estimate $(4.1)$ then follows from the hypothesis on $\Phi$, proving Lemma 4.2.2. 
Returning to $I$, we see that the hypothesis of Lemma 4.2 .2 is satisfied with $A \lesssim\left(\rho_{1} \rho_{2}\right)^{-1}$ by property (II-3) (applied to $T^{*}$ instead of $T$, where the $\varphi_{j}$ 's are supported in balls $B_{\rho_{j}}^{j}$ ). Thus

$$
|I| \lesssim \frac{r}{\rho_{1}} \frac{r_{2}}{\rho_{2}} \int_{B_{r_{1}}\left(\bar{y}_{1}\right)} \int_{B_{r_{2}}\left(\bar{y}_{2}\right)} d y_{1} d y_{2}=\frac{r_{1}}{\rho_{1}} \frac{r_{2}}{\rho_{2}} V_{r_{1}}^{1}\left(\bar{y}_{1}\right) V_{r_{2}}^{2}\left(\bar{y}_{2}\right)
$$

and conclusion (a) is established.

Proof of (b):

By (II-2),

$$
I=\int_{M_{2}}\left\langle T^{\varphi_{2}, x_{2}} \varphi_{1}, \psi_{1}\right\rangle \psi_{2}\left(x_{2}\right) d x_{2}
$$

Since $\int \psi_{2}\left(x_{2}\right) d x_{2}=0$, we see that I equals

$$
\int_{M_{2}}\left(\left\langle T^{\varphi_{2}, x_{2}} \varphi_{1}, \psi_{1}\right\rangle-\left\langle T^{\varphi_{2}, \bar{x}_{2}} \varphi_{1}, \psi_{1}\right\rangle\right) \psi_{2}\left(x_{2}\right) d x_{2}
$$

where $\bar{x}_{2}$ is the center of the ball associated to $\psi_{2}$, (which has radius $r_{2}$ ). The difference $T^{\varphi_{2}, x_{2}}-T^{\varphi_{2}, \bar{x}_{2}}$ leads to a factor $O\left(r_{2} \cdot \rho_{2}^{-1}\right)$ multiplied by the factor

$$
\frac{r_{1}}{d\left(\bar{x}_{1}, \bar{y}_{1}\right)} \frac{V_{\rho_{1}}\left(\bar{x}_{1}\right) \cdot V_{r_{1}}\left(\bar{y}_{1}\right)}{V\left(\bar{x}_{1}, \bar{y}_{1}\right)}
$$

coming from the estimate (b) in Observation 1. Taking into account the volume of integration of the ball $B_{2}$ we finally get as an estimate for $|I|$ that it is bounded by a multiple of

$$
\frac{r_{2}}{\rho_{2}} V_{r_{2}}^{2}\left(\bar{y}_{2}\right) \cdot \frac{r_{1}}{d_{1}\left(\bar{x}_{1}, \bar{y}_{1}\right)} \frac{V_{\rho_{1}}^{1}\left(\bar{x}_{1}\right) V_{r_{1}}^{1}\left(\bar{y}_{1}\right)}{V\left(\bar{x}_{1}, \bar{y}_{2}\right)}
$$

and (b) is proved.

\section{Proof of $(\mathrm{c})$ :}

Because the supports of $\psi_{r_{1}}$ and $\varphi_{\rho_{1}}$ are disjoint, as well as the supports of $\psi_{r_{2}}$ and $\varphi_{\rho_{2}}$, we have by (II- 1 )

$$
I=\int K\left(x_{1}, y_{1} ; x_{2}, y_{2}\right) \psi_{r_{1}}\left(y_{1}\right) \psi_{r_{2}}\left(y_{2}\right) \varphi_{\rho_{1}}\left(x_{1}\right) \varphi_{\rho_{2}}\left(x_{2}\right) d x_{1} d x_{2} d y_{1} d y_{2}
$$

In $I$ we replace $K=K\left(\cdot, y_{1} ; \cdot y_{2}\right)$ by

$$
K\left(\cdot, y_{1} ; \cdot, y_{2}\right)-K\left(\cdot, y_{1} ; \cdot, \bar{y}_{2}\right)-K\left(\cdot, \bar{y}_{1} ; \cdot, y_{2}\right)-K\left(\cdot, \bar{y}_{1} ; \cdot, \bar{y}_{2}\right) .
$$


Applying the lemma above we see that this double-difference is

$$
O\left(r_{1} \cdot r_{2}\left(d_{1} V_{1}\right)^{-1} \cdot\left(d_{2} V_{2}\right)^{-1}\right)
$$

in this range, where $d_{j} V_{j}=d_{j}\left(\bar{x}_{j}, \bar{y}_{j}\right) V_{j}\left(d_{j}\left(\bar{x}_{j}, \bar{y}_{j}\right)\right)$, since

$$
\left|D_{y_{1}} D_{y_{2}} K\left(\cdot y_{1} ; \cdot y_{2}\right)\right| \lesssim \frac{1}{\left(d_{1} V_{1}\right)} \cdot \frac{1}{\left(d_{2} V_{2}\right)}
$$

in this range. An integration then gives the desired conclusion, and completes the proof of Observation 4.2.1.

The analogue of Observation 3.4.2 in Subsection 3.4 now follows immediately from this. We replace the bump functions $\varphi_{\rho_{1}}, \varphi_{\rho_{2}}, \psi_{r_{1}}, \psi_{r_{2}}$ by their adjusted variants i.e.

$$
\varphi_{\rho_{j}}^{*}=\frac{\varphi_{\rho_{j}}}{V_{\bar{x}_{j}}^{j}\left(\rho_{j}\right)}, \quad \psi_{r_{j}}^{*}=\frac{\psi_{r_{j}}}{V_{\bar{y}_{j}}^{j}\left(r_{j}\right)}
$$

and setting

$$
I^{*}=\left\langle T\left(\psi_{r_{1}}^{*} \otimes \psi_{r_{2}}^{*}\right), \varphi_{\rho_{1}}^{*} \otimes \varphi_{\rho_{2}}^{*}\right\rangle .
$$

Observation 4.2.3 Assume $\rho_{1} \geq r_{1}, \rho_{2} \geq r_{2}$

(a) For all values of the parameters we have

$$
\left|I^{*}\right| \leq \frac{r_{1}}{\rho_{1}} \frac{r_{2}}{\rho_{2}} \frac{1}{V_{\bar{x}_{1}}^{1}\left(\rho_{1}\right) V_{\bar{x}_{2}}^{2}\left(\rho_{2}\right)}
$$

(b) If $d_{1}\left(\bar{x}_{1}, \bar{y}_{1}\right) \geq 10 \rho$, then

$$
\left|I^{*}\right| \leq \frac{r_{2}}{\rho_{2}} \frac{1}{V_{\bar{x}_{2}}^{2}\left(\rho_{2}\right)} \cdot \frac{r_{1}}{d_{1}\left(\bar{x}_{1}, \bar{y}_{1}\right)} \frac{1}{V_{\bar{x}_{1}}\left(d_{1}\left(\bar{x}_{1}, \bar{y}_{1}\right)\right)}
$$

(c) If $d_{1}\left(\bar{x}_{1}, \bar{y}\right) \geq 10 \rho_{1}$ and $d_{2}\left(\bar{x}_{2}, \bar{y}_{2}\right) \geq 10 \rho_{2}$, then

$$
\left|I^{*}\right| \leq \frac{r_{1} r_{2}}{d_{1}\left(\bar{x}_{1}, \bar{y}_{1}\right) d_{2}\left(\bar{x}_{2}, \bar{y}_{2}\right)} \cdot \frac{1}{V_{\bar{x}_{1}}\left(d_{1},\left(\bar{x}_{1}, \bar{y}_{1}\right)\right) V_{\bar{x}_{2}}\left(d_{2}\left(\bar{x}_{2}, \bar{y}_{2}\right)\right)}
$$

These observations lead directly as in Section 3 to the proof of the analogue of Proposition 3.3.1.

Proposition 4.2.4 Suppose $T$ is a product singular integral satisfying II-1 to II-6. Then

$$
\left|Q_{t_{1}}^{1} \cdot Q_{t_{2}}^{2} T Q_{s_{1}}^{1} \cdot Q_{s_{2}} f\right| \lesssim\left(\frac{t_{1}}{s_{1}} \cdot \frac{t_{2}}{s_{2}}\right)^{1 / 2} \mathcal{M}_{1} \mathcal{M}_{2}(f),
$$

if $t_{1} \leq s_{1}$ and $t_{2} \leq s_{2}$. Here $\mathcal{M}_{1}$ and $\mathcal{M}_{2}$ are the usual maximal functions on $M_{1}$ and $M_{2}$ respectively. There are similar inequalities if $s_{1} \leq t_{1}$ and/or $s_{2} \leq t_{2}$. 


\subsection{Proof of Theorem 4.1.2}

We shall now abbreviate by writing

$$
\widetilde{Q}_{s}=Q_{s_{1}}^{1} \cdot Q_{s_{2}}^{2}
$$

Then we have that

$$
\widetilde{Q}_{t} T=\int_{0}^{\infty} \int_{0}^{\infty} \widetilde{Q}_{t} T \widetilde{Q}_{s}^{2} \frac{d s_{1} d s_{2}}{s_{1} \cdot s_{2}} .
$$

Hence, arguing as before

$$
\left|\widetilde{Q}_{t} T(f)\right| \lesssim \int_{0}^{\infty} \int_{0}^{\infty} m(s, t) \mathcal{M}_{1} \mathcal{M}_{2} \widetilde{Q}_{s}(f) \frac{d s_{1} d s_{2}}{s_{1} \cdot s_{2}}
$$

where $m(s, t)=\min \left(\frac{t_{1}}{s_{1}}, \frac{s_{1}}{t_{1}}\right)^{1 / 2} \cdot \min \left(\frac{t_{2}}{s_{2}}, \frac{s_{2}}{t_{2}}\right)^{1 / 2}$.

We then have the following two-dimensional version of Hardy's inequality: if

$$
F(t)=\int_{0}^{\infty} \int_{0}^{\infty} m(t, s) G(s) \frac{d s_{1} d s_{2}}{s_{1} \cdot s_{2}}
$$

then

$$
\int_{0}^{\infty} \int_{0}^{\infty}|F(t)|^{2} \frac{d t_{1} d t_{2}}{t_{1} t_{2}} \lesssim \int_{0}^{\infty} \int_{0}^{\infty}|G(s)|^{2} \frac{d s_{1} d s_{2}}{s_{1} \cdot s_{2}}
$$

Applying this we get that

$$
\int_{0}^{\infty} \int_{0}^{\infty}\left|\widetilde{Q}_{t}(T f)\right|^{2} \frac{d t_{1} d t_{2}}{t_{1} t_{2}} \lesssim \int_{0}^{\infty} \int_{0}^{\infty}\left|\mathcal{M}_{1} \mathcal{M}_{2}(f)\right|^{2} \frac{d s_{1} d s_{2}}{s_{1} s_{2}}
$$

and invoking Proposition 2.6.1 we will have completed the proof Theorem 4.1 .2 once we verify that

$$
\left\|E_{0}^{i}(T f)\right\|_{L^{p}(\widetilde{M})} \lesssim\|f\|_{L^{p}(\widetilde{M})}, \quad i=1,2,
$$

for $1<p<\infty$.

To prove (4.2) note that if $f \in C_{0}^{\infty}(\widetilde{M})$, then

$$
E_{0}^{2}(f)\left(y_{1}, y_{2}\right)=F_{1}\left(y_{1}\right) \otimes 1, \text { where } F_{1}\left(y_{1}\right)=c \int_{M_{2}} f\left(y_{1}, y_{2}\right) d y_{2} .
$$

and $c=\left|M_{1}\right|^{-1}$. We claim that

$$
T E_{0}^{2}(f)\left(x_{1}, x_{2}\right)=T^{1, x_{2}}\left(F_{1}\right)\left(x_{1}\right) .
$$

In fact

$$
\left\langle T E_{0}^{2}(f) \psi_{1} \otimes \psi_{2}\right\rangle=\left\langle T\left(F_{1} \otimes 1\right), \psi_{1} \otimes \psi_{2}\right\rangle=\int_{M_{2}}\left\langle T^{1, x_{2}}\left(f_{1}\right), \psi_{1}\right\rangle \psi_{2}\left(x_{2}\right) d x_{2},
$$

by property II-2, if we take $\varphi_{1}=F_{1}, \varphi_{2}=1$. 
This means that both sides of (4.3) have the same value when tested against $\psi_{1} \otimes \psi_{2}$. Since $\psi_{1}$ and $\psi_{2}$ are arbitrary bump functions, (4.3) is established, and from this it follows by the one-factor theory that $T E_{0}^{2}$ is bounded on $L^{p}$. Using duality, and the fact that the class of product operators is self-dual then proves (4.2). Similarly, the analogue with $E_{0}^{2}$ replaced by $E_{0}^{1}$ also holds, and Theorem 4.1.2 is proved.

\section{The case of $n$ factors}

We now consider the case when there are $n$ factors, $\widetilde{M}=M_{1} \times \cdots \times M_{n}$. To define the class of singular integrals on $\widetilde{M}$ we proceed inductively, assuming we have already done so in the case of $n-1$ factor. The passage from $n-1$ to $n$ will be completely analogous to the passage from 1 factor to 2 factors carried out above.

We assume that $T$ is a linear mapping from $C_{0}^{\infty}(\widetilde{M})$ to $C^{\infty}(\widetilde{M})$, which has an associated distribution kernel $K(x, y),(x \in \widetilde{M}, y \in \widetilde{M})$, which is $C^{\infty}$ away from the cross $=\left\{(x, y) ; x_{j}=y_{j}\right.$, for some $\left.j, 1 \leq j \leq n\right\}$. We assume also

$$
\begin{aligned}
& \left\langle T\left(\varphi_{1} \otimes \cdots \otimes \varphi_{n}\right), \psi_{1} \otimes \psi_{2} \cdots \otimes \psi_{n}\right\rangle= \\
& =\int K(x, y) \prod_{i=1}^{n} \varphi_{i}\left(x_{i}\right) \psi_{i}\left(y_{i}\right) d x d y
\end{aligned}
$$

whenever $\varphi_{i}$ and $\psi_{i}$ are bump-functions on $M_{i}$, with disjoint supports, for $1 \leq i \leq n$.

(III-2) For each bump function $\varphi_{n}$ on $M_{n}$, and each $x_{n} \in M_{n}$, there is a singular integral $T^{\varphi_{n}, x_{n}}$ (of the $n-1$ factor type) on $M_{1} \times \cdots \times M_{n-1}$, so that $x_{n} \longrightarrow T^{\varphi_{n}, x_{n}}$ is smooth in the sense below and so that

$$
\begin{gathered}
\left\langle T\left(\varphi_{1} \otimes \cdots \otimes \varphi_{n}\right), \psi_{1} \otimes \cdots \otimes \psi_{n}\right\rangle= \\
=\int_{M_{n}}\left\langle T^{\varphi_{n}, x_{n}}\left(\varphi_{1} \otimes \cdots \otimes \varphi_{n-1}\right), \psi_{1} \otimes \cdots \otimes \psi_{n-1}\right\rangle \psi_{n}\left(x_{n}\right) d x_{n} .
\end{gathered}
$$

Moreover, we require that $T^{\varphi_{n}, x_{n}}$ satisfy the conditions for $M_{n-1}$ factors uniformly, as well as $\rho_{n}^{L} \partial_{X_{n}}^{L}\left(T^{\varphi_{n}, x_{n}}\right)$, for each $L$, when $\rho_{n}$ is the radius associated to $\varphi_{n}$.

$$
\prod_{j=1}^{n}\left(\partial_{X_{j}}^{a_{j}} T\left(\varphi_{1} \otimes \cdots \otimes \varphi_{j}\right) \lesssim \prod_{j=1}^{n} r_{j}^{-a_{j}}\right.
$$

where $r_{j}$ are the radii of the balls associated to the $\varphi_{j}$. In (III-2) and (III-3) the inequalities are taken in the sense of (I-2). 
(III-4)

$$
\left|\prod_{j=1}^{n}\left(\partial_{X_{j}, Y_{j}}^{a_{j}}\right) K(x, y)\right| \lesssim \prod_{j=1}^{n} \frac{d_{j}\left(x_{j}, y_{j}\right)^{-1}}{V_{j}\left(x_{j}, y_{j}\right)}
$$

(III-5) The same conditions hold for any permutation of the indices $1, \ldots, n$.

(III-6) The same properties are assumed for all $2^{n}-1$ of the "transposes" of $T$ which arise whenever we interchange the $x_{j}$ with the $y_{j}$, for (some) of the $j$.

\subsection{The main theorem}

In complete analogy with Theorem 3.1, we then have:

Theorem 5.1.1 Each product singular integral on $\widetilde{M}=M_{1} \times \cdots \times M_{n}$ satisfying conditions (III-1) to (III-6) extends to a bounded operator on $L^{p}(\widetilde{M})$ to itself, whenever $1<p<\infty$.

\section{References}

[CNS92] Chang, D.-C., Nagel, A. and Stein, E. M.: Estimates for the $\bar{\partial}$-Neumann problem in pseudoconvex domains of finite type in $\mathbb{C}^{2}$. Acta Math. 169 (1992), 153-228.

[DJ84] David, G. And Journé, J.-L.: A boundedness criterion for generalized Calderón-Zygmund operators. Ann. of Math.(2) 120 (1984), 371397.

[FS82] Fefferman, R. And Stein, E.M.: Singular integrals on product spaces. Adv. in Math. 45 (1982), 117-143.

[JMZ35] Jessen, B., Marcinkiewicz, J. And Zygmund, A.: Note on the differentiability of multiple integrals. Fund. Math. 25 (1935), 217-234.

[Jou85] Journé, J.L.: Calderón-Zygmund operators on product spaces. Rev. Mat. Iberoamericana 1 (1985), 55-91.

[Koe02] Koenig, K.: On maximal Sobolev and Hölder estimates for the tangential Cauchy-Riemann operator and boundary Laplacian. Amer. J. Math. 124 (2002), 129-197.

[MRS95] MÜller, D., Ricci, F. AND Stein, E.M.: Marcinkiewicz multipliers and multi-parameter structure on Heisenberg (-type) groups, I. Invent. Math. 119 (1995), 119-233.

[NRS01] Nagel, A., Ricci, F. And Stein, E.M.: Singular integrals with flag kernels and analysis on quadratic CR manifolds. J. Funct. Anal. 181 (2001), 29-118.

[NRSW89] Nagel, A., Rosay, J.-P., Stein, E.M. and Wainger, S.: Estimates for the Bergman and Szegö kernels in $\mathbb{C}^{2}$. Ann. of Math. 129 (1989), 113-149. 
[NS01a] Nagel, A. And Stein, E.M.: The $\square_{b}$-Heat equation on pseudoconvex manifolds of finite type in $\mathbb{C}^{2}$. Math. Z. 238 (2001), 37-88.

[NS01b] Nagel, A. And Stein, E.M.: Differentiable control metrics and scaled bump functions. J. Differential Geom. 57 (2001), 465-492.

[NS03] Nagel, A. And Stein, E.M.: The $\bar{\partial}_{b}$-complex on decoupled boundaries in $\mathbb{C}^{n}$. Preprint.

[NSW85] Nagel, A., Stein, E.M. And Wainger. S.: Balls and metrics defined by vector fields I. Basic properties. Acta Math. 155 (1985), 103-147.

[Ste70] Stein, E.M.: Topics in harmonic analysis related to the LittlewoodPaley theory. Annals of Mathematics Studies 63. Princeton University Press, Princeton, New Jersey, 1970.

[Ste93] Stein, E.M.: Harmonic analysis: real-variable methods, orthogonality, and oscillatory integrals. Princeton Mathematical Series 43. Monographs in Harmonic Analysis, III. Princeton University Press, Princeton, NJ, 1993

Recibido: 27 de septiembre de 2002

\author{
Alexander Nagel \\ Department of Mathematics \\ University of Wisconsin \\ Madison, WI 53706-1388, USA \\ nagel@math.wisc.edu \\ Elias M. Stein \\ Department of Mathematics \\ Princeton University \\ Princeton, NJ 08544, USA \\ stein@math.princeton.edu
}

Supported in part by grants from the National Science Foundation. 